\title{
Automated Simulation Updates based on Flight Data
}

\author{
Eugene A. Morelli ${ }^{1}$ \\ NASA Langley Research Center, Hampton, VA, 23681 \\ David G. Ward ${ }^{2}$ \\ Barron Associates, Inc., Charlottesville, VA, 22901
}

\begin{abstract}
A statistically-based method for using flight data to update aerodynamic data tables used in flight simulators is explained and demonstrated. A simplified wind-tunnel aerodynamic database for the F/A-18 aircraft is used as a starting point. Flight data from the NASA F-18 High Alpha Research Vehicle (HARV) is then used to update the data tables so that the resulting aerodynamic model characterizes the aerodynamics of the F-18 HARV. Prediction cases are used to show the effectiveness of the automated method, which requires no ad hoc adjustments by the analyst.
\end{abstract}

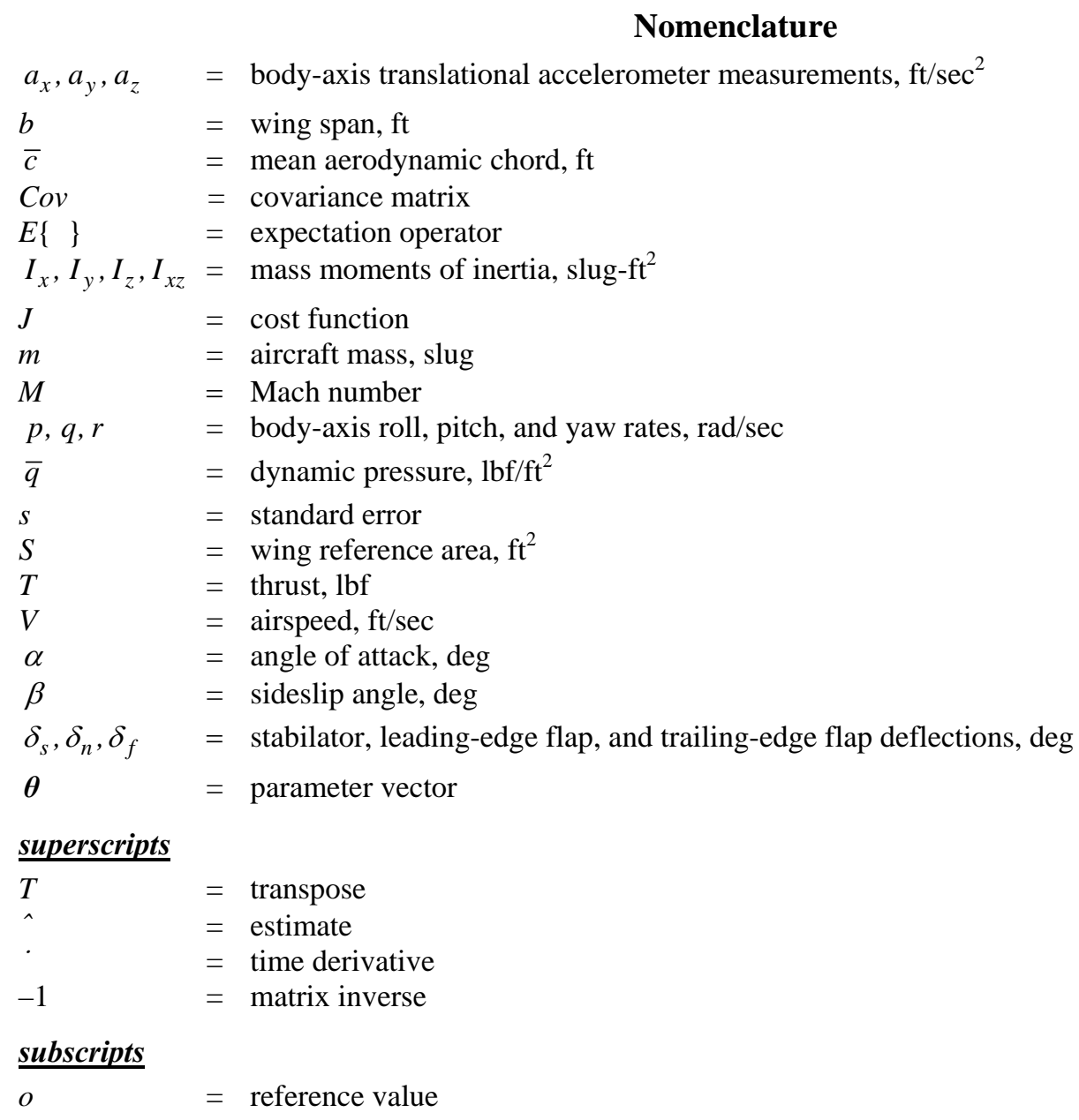

\footnotetext{
${ }^{1}$ Research Engineer, Dynamic Systems and Control Branch, MS 308, Associate Fellow.

${ }^{2}$ Research Scientist, 1410 Sachem Place, Suite 202, Associate Fellow.
} 


\section{Introduction}

$\mathrm{A}$ IRCRAFT research, development, and testing programs require high-fidelity simulations and aerodynamic models. As an aircraft is readied for flight testing, considerable effort is spent in developing aerodynamic models based on tabular data obtained from wind-tunnel tests. Updating these wind-tunnel-derived aerodynamic models using flight data is important because wind-tunnel data is not fully representative of the full-scale aircraft, due to effects such as Reynolds number differences, wind-tunnel model geometry differences, wind-tunnel sting effects, flow angularity and blockage in the wind tunnel, inability to test in the wind-tunnel at certain flight conditions and vehicle configurations, and wind-tunnel wall effects, among others.

The current state-of-the-art for updating a simulation aerodynamic database from flight data involves time-intensive and $a d$ hoc adjustments to individual values in the aerodynamic data tables to improve the match between flight data and corresponding quantities computed from the simulation. Another approach is to use system identification methods to identify increments that can be added to the original wind-tunnel database to improve the match between flight data and the simulation. The problems with these approaches are: 1) a large amount of time and judgment is required from a human analyst, and 2) the updates may work well for the individual maneuver(s) on which the update was based, but the updated simulation may predict poorly for other maneuvers. Because of the cost and technical challenges associated with updating simulation databases, such updating is frequently not done.

In this paper, a statistically-based method is developed for automatically identifying updates to aerodynamic data tables, based on flight data. The approach involves localized multivariate polynomial modeling of the aerodynamic data tables, and the use of these local models as a priori information for the flight data analysis. In effect, the information in the aerodynamic data tables, which is diffused over many data table values, is converted to a parametric model in the local subspace corresponding to the flight maneuver. Because of the large number of variables that influence the aerodynamics, these local models are hyper-surfaces. The local hyper-surface model provides a valid basis for the model structure and parameter values, which are used as a priori information for the flight data analysis. If the flight data have information content relative to any of the local hyper-surface model parameters, those model parameters are modified to best fit the information in both the a priori hyper-surface model and the flight data, using statistical weighting. If there is no information in the flight data relative to a given model parameter, then that hyper-surface model parameter remains unchanged and equal to its a priori value. The result is a modified local hyper-surface model that gives the best characterization of the local multivariate functional dependencies, based on all the available data. All of this is done in an automated fashion, using statistical measures of parameter accuracy, so there is no need for the analyst to make ad hoc adjustments to the data tables or the procedure.

Following the update, the modified surface model is smoothed at the edges of the local subspace where it is valid, to avoid discontinuities with adjacent parts of the aerodynamic database that have not been updated using flight data. This is done using a multivariate Gaussian blending function to retain continuity of magnitudes and derivatives at the edges. Finally, the aerodynamic database can be re-populated using the updated aerodynamic model, or the updates can be recorded as increments.

The paper is organized as follows. First, background and theory are introduced, and the algorithms for the flight updates and edge smoothing are developed. Then a realistic example is given, where a simplified wind-tunnel aerodynamic database for the F/A-18 aircraft is modified using flight data from the F-18 High Alpha Research Vehicle (HARV), so that the resulting aerodynamic model characterizes the F-18 HARV. Prediction maneuvers are used to validate that the updated aerodynamic model accurately characterizes the F-18 HARV aerodynamics.

\section{Method}

\section{A. Response Surface Modeling}

The procedure begins with a local hyper-surface model, also called a response surface model, based on the aerodynamic data tables in the simulation. The aerodynamic database is interrogated to obtain values of the non-dimensional force and moment coefficients for various settings of the variables that influence the aerodynamics. The non-dimensional force and moment coefficients represent response variables, and the variables that influence the response variables (usually non-dimensional aircraft states and control surface deflections) are called explanatory variables. The intent is to collect sufficient data for local hyper-surface modeling. The ranges of explanatory variables used for this data collection are defined by the ranges of the those variables observed during the flight test maneuver(s) that will be used for the update. An example would be to interrogate the aerodynamic database for values of lift coefficient as the angle of attack, Mach number, pitch rate, and stabilator deflection vary over the range of values observed in a flight test maneuver. The data points collected from the aerodynamic 
database are specified as actual data points from the wind tunnel database, rather than interpolated points. This is done so that the proper statistical weighting is implemented when the local hyper-surface model is updated based on flight data. If many interpolated points are included when collecting data from the wind tunnel database for local hyper-surface modeling, then the uncertainty of the local hyper-surface model will be artificially reduced, and the flight data will not be given proper statistical weighting.

Once the data is collected by interrogating the aerodynamic database, the local response surface modeling is done using multivariate orthogonal functions ${ }^{1-3}$. In this method, arbitrary functions of the explanatory variables, usually specified as multivariate polynomials, are generated first. These functions are then orthogonalized using a Gram-Schmidt orthogonalization procedure, which will be described later.

The form of the model to be identified is

$$
\boldsymbol{z}=a_{1} \boldsymbol{p}_{1}+a_{2} \boldsymbol{p}_{2}+\ldots+a_{n} \boldsymbol{p}_{n}+\boldsymbol{\varepsilon}
$$

where $z$ is an $N$-dimensional vector of measured response variable values, $z=\left[z_{1}, z_{2}, \ldots, z_{N}\right]^{T}$, modeled in terms of a linear combination of $n$ mutually orthogonal modeling functions $\boldsymbol{p}_{j}, j=1,2, \ldots, n$. Each $\boldsymbol{p}_{j}$ is an $N$-dimensional vector which in general depends on the explanatory variables. The $a_{j}, j=1,2, \ldots, n$ are constant model parameters to be determined, and $\boldsymbol{\varepsilon}$ denotes the modeling error vector.

Equation (1) represents a mathematical model used to fit a response surface to measured data. We put aside for the moment the important questions of determining how the modeling functions $\boldsymbol{p}_{j}$ are computed from the explanatory variables, as well as which modeling functions should be included in Eq. (1), which implicitly determines $n$. Now define an $N$ xn matrix $\boldsymbol{P}$,

$$
\boldsymbol{P}=\left[\boldsymbol{p}_{1}, \boldsymbol{p}_{2}, \ldots, \boldsymbol{p}_{n}\right]
$$

and let $\boldsymbol{a}=\left[a_{1}, a_{2}, \ldots, a_{n}\right]^{T}$. Eq. (1) can then be written as a standard least squares regression problem,

$$
z=P a+\varepsilon
$$

The variable $\varepsilon$ represents a vector of errors that are to be minimized in a least squares sense. The goal is to determine $\boldsymbol{a}$ that minimizes the least squares cost function

$$
J=\frac{1}{2}(z-P a)^{T}(z-P a)=\frac{1}{2} \varepsilon^{T} \varepsilon
$$

The parameter vector estimate $\hat{\boldsymbol{a}}$ that minimizes this cost function is computed as ${ }^{1}$

$$
\hat{\boldsymbol{a}}=\left[\boldsymbol{P}^{T} \boldsymbol{P}\right]^{-1} \boldsymbol{P}^{T} \boldsymbol{z}
$$

The estimated parameter covariance matrix is ${ }^{1}$

$$
\operatorname{Cov}(\hat{\boldsymbol{a}})=E\left[(\hat{\boldsymbol{a}}-\boldsymbol{a})(\hat{\boldsymbol{a}}-\boldsymbol{a})^{T}\right]=\sigma^{2}\left(\boldsymbol{P}^{T} \boldsymbol{P}\right)^{-1}
$$

where $E$ is the expectation operator, and the fit error variance $\sigma^{2}$ can be estimated from the residuals

$$
v=z-P \hat{a}
$$

using

$$
\hat{\sigma}^{2}=\frac{1}{(N-n)}\left[(z-P \hat{a})^{T}(z-P \hat{a})\right]=\frac{\boldsymbol{v}^{T} \boldsymbol{v}}{(N-n)}
$$


Parameter standard errors are computed as the square root of the diagonal elements of the $\operatorname{Cov}(\hat{\boldsymbol{a}})$ matrix from Eq. (6), using $\hat{\sigma}^{2}$ from Eq. (8). The identified model output $\boldsymbol{y}$ is computed as

$$
\boldsymbol{y}=\boldsymbol{P} \hat{\boldsymbol{a}}
$$

In conventional response surface modeling, the modeling functions (columns of $\boldsymbol{P}$ ) are often polynomials in the explanatory variables. This approach corresponds to using the terms of a multivariate Taylor series expansion to approximate the functional dependence of the response variable on the explanatory variables. If the modeling functions are instead multivariate orthogonal functions generated from the explanatory variable data, it is easier to determine an appropriate model structure for response surface modeling, because the explanatory capability of each modeling function is completely distinct from all the others. This decouples the least squares modeling problem, as will be shown now.

For mutually orthogonal modeling functions,

$$
\boldsymbol{p}_{i}^{T} \boldsymbol{p}_{j}=0 \quad, \quad i \neq j \quad, \quad i, j=1,2, \ldots, n
$$

and $\boldsymbol{P}^{T} \boldsymbol{P}$ is a diagonal matrix with the inner product of the orthogonal functions on the main diagonal. Using Eqs. (2) and (10) in Eq. (5), the $j^{\text {th }}$ element of the estimated parameter vector $\hat{\boldsymbol{a}}$ is given by

$$
\hat{a}_{j}=\left(\begin{array}{ll}
\boldsymbol{p}_{j}^{T} & \boldsymbol{z}
\end{array}\right) /\left(\begin{array}{ll}
\boldsymbol{p}_{j}^{T} & \boldsymbol{p}_{j}
\end{array}\right)
$$

Using Eqs. (2), (10), and (11) in Eq. (4),

$$
\hat{J}=\frac{1}{2}\left[\boldsymbol{z}^{T} \boldsymbol{z}-\sum_{j=1}^{n}\left(\boldsymbol{p}_{j}^{T} \boldsymbol{z}\right)^{2} /\left(\boldsymbol{p}_{j}^{T} \boldsymbol{p}_{j}\right)\right]
$$

Eq. (12) shows that when the modeling functions are orthogonal, the reduction in the estimated cost resulting from including the term $a_{j} \boldsymbol{p}_{j}$ in the model depends only on the response variable data $\boldsymbol{z}$ and the added orthogonal modeling function $\boldsymbol{p}_{j}$. This decouples the least squares modeling problem, and makes it possible to evaluate each orthogonal modeling function in terms of its ability to reduce the least squares model fit to the data, regardless of which other orthogonal modeling functions are already selected for the model. When the modeling functions $\boldsymbol{p}_{j}$ are instead polynomials in the explanatory variables (or any other non-orthogonal function set), the least squares problem is not decoupled, and iterative analysis is required to find the subset of modeling functions for an adequate model structure.

The orthogonal modeling functions to be included in the model are chosen to minimize predicted squared error, $P S E$, defined by $^{4}$

$$
P S E=\frac{(\boldsymbol{z}-\boldsymbol{P} \hat{\boldsymbol{a}})^{T}(\boldsymbol{z}-\boldsymbol{P} \hat{\boldsymbol{a}})}{N}+\sigma_{\max }^{2} \frac{n}{N}
$$

or

$$
P S E=\frac{2 \hat{J}}{N}+\sigma_{\max }^{2} \frac{n}{N}
$$

The constant $\sigma_{\max }^{2}$ is the a priori upper-bound estimate of the squared error between future data and the model, i.e., the upper bound mean squared error for prediction cases. The upper bound is used in the model over-fit penalty term to account for the fact that PSE is calculated when the model structure is not correct, i.e., during the model structure determination stage. Using the upper bound is conservative in the sense that model complexity will be 
minimized as a result of using an upper bound for this constant in the penalty term. Because of this, the value of PSE computed from Eq. (14) for a particular model structure tends to overestimate actual prediction errors on new data. Therefore, the PSE metric conservatively estimates the squared error for prediction cases.

A simple estimate of $\sigma_{\max }^{2}$ that is independent of the model structure can be obtained by considering $\sigma_{\max }^{2}$ to be the residual variance estimate for a constant model equal to the mean of the measured response values,

$$
\sigma_{\max }^{2}=\frac{1}{N} \sum_{i=1}^{N}\left[z_{i}-\bar{z}\right]^{2}
$$

where

$$
\bar{z}=\frac{1}{N} \sum_{i=1}^{N} z_{i}
$$

The PSE in Eq. (14) depends on the mean squared fit error $2 \hat{J} / N$, and a term proportional to the number of terms in the model, $n$. The latter term prevents over-fitting the data with too many model terms, which is detrimental to model prediction accuracy ${ }^{1,4}$. Note that while the mean squared fit error $2 \hat{J} / N$ must decrease with the addition of each orthogonal modeling function to the model (by Eq. (12)), the over-fit penalty term $\sigma_{\max }^{2} n / N$ increases with each added model term ( $n$ increases). Introducing the orthogonal modeling functions into the model in order of most effective to least effective in reducing the mean squared fit error (quantified by $\left(\boldsymbol{p}_{j}^{T} \boldsymbol{z}\right)^{2} /\left(\boldsymbol{p}_{j}^{T} \boldsymbol{p}_{j}\right)$ for the $j^{\text {th }}$ orthogonal modeling function) means that the PSE metric will always have a single global minimum. Figure 1 depicts this graphically, using actual modeling results from Ref. [5]. The figure shows that after the first 6 modeling functions, the added model complexity associated with an additional orthogonal modeling function is not justified by the associated reduction in mean squared fit error. This point is marked by a minimum PSE, which defines an adequate model structure with good predictive capability. Ref. [4]

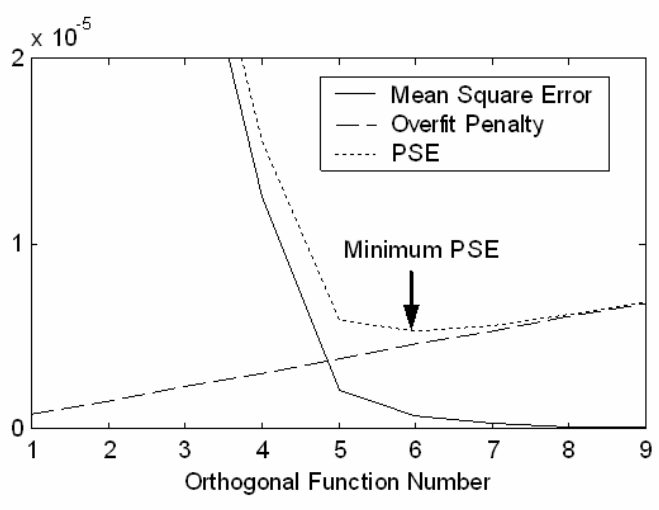

Figure 1. Model structure determination using orthogonal functions and $P S E$ contains further statistical arguments and analysis for the form of PSE given in Eq. (14), including justification for its use in modeling problems.

Using orthogonal functions to model the response variable makes it possible to evaluate the merit of including each modeling function individually, using the predicted squared error PSE. The goal is to select a model structure with minimum PSE, and the PSE always has a single global minimum for orthogonal modeling functions. This makes the model structure determination a well-defined and straightforward process that can be (and was) automated.

\section{B. Generating Orthogonal Modeling Functions}

Ref. [2] describes a procedure for using data for the explanatory variables to generate multivariate orthogonal modeling functions $\boldsymbol{p}_{j}, j=0,1,2, \ldots, n$, with the following important orthogonality property:

$$
\boldsymbol{p}_{i}^{T} \boldsymbol{p}_{j}=0 \quad i \neq j \quad i, j=1,2, \ldots, n
$$

It is also possible to generate multivariate orthogonal functions by first generating ordinary multivariate functions in the explanatory variables, then orthogonalizing these functions using a Gram-Schmidt orthogonalization procedure. This approach is described in Refs. [1] and [3], which are the basis for the material presented here. 
The process begins by choosing one of the ordinary multivariate functions as the first orthogonal function. Typically, a vector of ones (associated with the bias term in the model) is chosen as the first orthogonal function,

$$
p_{1}=1
$$

In general, any function of the explanatory variables can be chosen as the first orthogonal function, without any change in the procedure. To generate the next orthogonal function, an ordinary multivariate polynomial function is made orthogonal to the preceding orthogonal function(s). Define the $j^{\text {th }}$ orthogonal function $\boldsymbol{p}_{j}$ as

$$
\boldsymbol{p}_{j}=\boldsymbol{\xi}_{j}-\sum_{k=1}^{j-1} \gamma_{k j} \boldsymbol{p}_{k} \quad j=2,3, \ldots, n
$$

where $\xi_{j}$ is the $j^{\text {th }}$ ordinary multivariate function vector. Typically, each $\xi_{j}$ would be some ordinary polynomial function of the explanatory variables. The $\gamma_{k j}$ for $k=1,2, \ldots, j-1$ are scalars determined by multiplying both sides of Eq. (19) by $\boldsymbol{p}_{k}^{T}$, invoking the mutual orthogonality of the $\boldsymbol{p}_{k}, k=1,2, \ldots, j$, and solving for $\gamma_{k j}$

$$
\gamma_{k j}=\frac{\boldsymbol{p}_{k}^{T} \boldsymbol{\xi}_{j}}{\boldsymbol{p}_{k}^{T} \boldsymbol{p}_{k}} \quad k=1,2, \ldots, j-1
$$

The same process can be implemented in sequence for each ordinary multivariate function $\xi_{j}, j=2,3, \ldots, n$. The total number of ordinary multivariate functions used as raw material for generating the multivariate orthogonal functions, including the bias term, is $n$. It can be seen from Eqs. (18)-(20) that each orthogonal function can be expressed exactly in terms of a linear expansion of the original multivariate functions. The orthogonal functions are generated sequentially by orthogonalizing the original multivariate functions with respect to the orthogonal functions already computed, so that each orthogonal function can be considered an orthogonalized version of an original multivariate function.

The orthogonalization process described above can be used to generate orthogonal functions derived from multivariate polynomials of arbitrary order in the explanatory variables, subject only to limitations related to the information contained in the data. For example, it is not possible to generate an orthogonal function corresponding to $\alpha^{2}$ if there are only two distinct values of angle of attack in the data. This is analogous to the requirement that at least three data points are needed to identify a quadratic model, which has three parameters. The same limit also applies to the orthogonal function corresponding to any cross term, such as $\alpha^{2} \delta_{s}$ for this example.

If the $\boldsymbol{p}_{j}$ vectors and the $\boldsymbol{\xi}_{j}$ vectors are arranged as columns of matrices $\boldsymbol{P}$ and $\boldsymbol{X}$, respectively, and the $\gamma_{k j}$ are elements in the $k^{\text {th }}$ row and $j^{\text {th }}$ column of an upper triangular matrix $\boldsymbol{G}$ with ones on the diagonal,

$$
\boldsymbol{G}=\left[\begin{array}{ccccc}
1 & \gamma_{12} & \gamma_{13} & \cdots & \gamma_{1 n} \\
0 & 1 & \gamma_{23} & \cdots & \gamma_{2 n} \\
0 & 0 & 1 & \cdots & \gamma_{3 n} \\
\vdots & \vdots & \vdots & \vdots & \vdots \\
0 & 0 & 0 & \cdots & 1
\end{array}\right]
$$

Then

$$
\boldsymbol{X}=\boldsymbol{P} \boldsymbol{G}
$$

which leads to 


$$
\boldsymbol{P}=\boldsymbol{X} \boldsymbol{G}^{-1}
$$

The columns of $\boldsymbol{G}^{-1}$ contain the coefficients for expansion of each column of $\boldsymbol{P}$ (i.e., each multivariate orthogonal function) in terms of an exact linear expansion in the original multivariate functions in the columns of $\boldsymbol{X}$. The manner in which the orthogonal functions are generated allows them to be decomposed without ambiguity into an expansion of the original multivariate functions, which have physical meaning.

The method described here is simple, and works regardless of the spacing for the explanatory data; i.e., the data do not have to be collected with uniform spacing of the explanatory variables on a rectangular grid.

\section{Conversion to physically-meaningful multivariate models}

After the model structure is determined using multivariate orthogonal modeling functions for minimum PSE, the identified model output is

$$
\boldsymbol{y}=\boldsymbol{P} \hat{a}
$$

where the $\boldsymbol{P}$ matrix now includes only the orthogonal functions selected in the model structure determination. Then, each retained orthogonal modeling function can be decomposed without error into an expansion of the original multivariate functions in the explanatory variables, using the columns of $\boldsymbol{G}^{-1}$ in Eq. (23) corresponding to the retained orthogonal functions. Common terms are combined using double precision arithmetic to arrive finally at a model using only original multivariate functions in the explanatory variables. Terms that contribute less than 0.1 percent of the final model root-mean-square magnitude are dropped. Assuming the original multivariate functions are polynomials in the explanatory variables, combining like terms in this final step puts the final model in the form of selected terms from a multivariate Taylor series expansion.

Although there are other methods available to do the response modeling described here, this multivariate orthogonal function method has been found to work well for modeling aerodynamic data tables for aircraft ${ }^{6,7}$, and can produce a final model comprised of ordinary multivariate polynomial terms. This model form is compatible with standard parameter estimation methods used for flight data analysis and modeling.

\section{Flight data analysis and modeling}

Once the response surface model has been identified, the result is a multivariate ordinary polynomial model, which can be considered a multivariate Taylor series for the response variable (i.e., a non-dimensional force or moment coefficient) in terms of the explanatory variables (e.g., angle of attack, non-dimensional pitch rate, control surface deflections, etc.). The model includes only the terms necessary to model the variation embodied in the aerodynamic data tables over the local subspace defined by the range of explanatory variables observed in the flight maneuver. Each parameter in the model has an estimated value and uncertainty bound, because the model was identified from data extracted from the aerodynamic data tables using a statistically-based least squares method, described earlier.

Now the flight data is introduced into the modeling problem. The identified local response surface model provides the a priori model structure and model parameter values for the flight data analysis. This is an important point, because generally the values that the explanatory variables take during a flight maneuver do not completely or systematically cover the local subspace of explanatory variables. More commonly, during a flight maneuver, the explanatory variables take a narrow trajectory through the local subspace of explanatory variables. Consequently, the flight data are ill-suited for determining the model structure needed to properly characterize the local topology of the underlying functional dependence. The aerodynamic data tables, however, can be interrogated to obtain good data, usually on a rectangular hyper-grid, which allows very accurate local response surface modeling. This is possible because wind tunnel testing typically includes comprehensive and independent variations of the explanatory variables. The flight data analysis treats the local response surface model based on the aerodynamic data tables as the $a$ priori model, and proceeds with the modeling using standard least squares with $a$ priori information.

The least squares cost function for analyzing flight data can include information from an a priori model using a Bayesian formulation, as follows ${ }^{1}$

$$
J(\theta)=\frac{1}{2 \sigma^{2}}(z-X \boldsymbol{\theta})^{T}(z-X \boldsymbol{\theta})+\frac{1}{2}\left(\boldsymbol{\theta}-\boldsymbol{\theta}_{p}\right)^{T} \Sigma_{p}^{-1}\left(\boldsymbol{\theta}-\boldsymbol{\theta}_{p}\right)
$$


where $\boldsymbol{\theta}_{p}$ is the parameter vector estimate for the a priori model, and $\Sigma_{p}$ is the associated covariance matrix. These quantities are computed using Eqs. (5)-(8), with $\boldsymbol{\theta}_{p}$ replacing $\hat{\boldsymbol{a}}$ in the notation. The parameter estimation results in this case are obtained from ${ }^{1,8}$

$$
\hat{\boldsymbol{\theta}}=\left[\left(\boldsymbol{X}^{T} \boldsymbol{X}\right) / \sigma^{2}+\Sigma_{p}^{-1}\right]^{-1}\left[\left(\boldsymbol{X}^{T} \boldsymbol{z}\right) / \sigma^{2}+\boldsymbol{\Sigma}_{p}^{-1} \boldsymbol{\theta}_{p}\right]
$$

where the fit error variance $\sigma^{2}$ for the flight data can be estimated from

$$
\hat{\sigma}^{2}=\frac{1}{(N-n)}\left[\left(z-\boldsymbol{X} \hat{\boldsymbol{\theta}}^{\prime}\right)^{T}\left(z-\boldsymbol{X} \hat{\boldsymbol{\theta}}^{\prime}\right)\right]
$$

and $\hat{\boldsymbol{\theta}}^{\prime}$ is computed based on the flight data alone,

$$
\hat{\boldsymbol{\theta}}^{\prime}=\left(\boldsymbol{X}^{T} \boldsymbol{X}\right)^{-1}\left(\boldsymbol{X}^{T} \boldsymbol{z}\right)
$$

The estimated parameter covariance matrix is ${ }^{1,8}$

$$
\operatorname{Cov}(\hat{\boldsymbol{\theta}})=\left[\left(\boldsymbol{X}^{T} \boldsymbol{X}\right) / \hat{\sigma}^{2}+\boldsymbol{\Sigma}_{p}^{-1}\right]^{-1}
$$

The expression for the flight-updated parameter estimate in Eq. (26) shows how the information content from the a priori model, quantified by the inverse covariance matrix $\Sigma_{p}^{-1}$, is balanced with the information content in the flight data, represented by $\left(\boldsymbol{X}^{T} \boldsymbol{X}\right) / \sigma^{2}$. For example, if the a priori estimate of a particular parameter is highly accurate (small value of the corresponding diagonal element of $\Sigma_{p}$ ), then there will have to be significant information related to that parameter in the flight data to move the estimate of that parameter from the a priori value. Similarly, any significant information in the quantity $\left(\boldsymbol{X}^{T} \boldsymbol{X}\right) / \sigma^{2}$ related to a particular parameter will overwhelm a priori information for that parameter, moving the parameter estimate toward the value that would have been computed based on the flight data alone. In a limiting case where, for example, there is no movement at all in a particular explanatory variable in the flight data, such as a trailing-edge flap, then the algorithm will retain the parameter estimate for the trailing-edge flap effectiveness that was identified for the a priori model based on the aerodynamic data tables. To ensure that the model updates are done properly, the reference values of the explanatory variables for the data collected from the aerodynamic database should be chosen the same as for the flight data. Typically, these reference values are obtained from flight data initial conditions or mean values.

Using this method, the balance between information derived from the a priori model based on the aerodynamic data tables and the information embodied in the flight data is done automatically for multiple parameters at once, based on statistical principles.

\section{E. Blending the flight updates}

After the local response surface model has been modified using flight data, the resulting hyper-surface may introduce discontinuities in magnitude and/or slope at the subspace boundaries. This is the result of the flight update being done on a subspace of the explanatory variables associated with a specific flight maneuver or set of flight maneuvers. To blend the updated response surface model with the surrounding aerodynamic database, a Gaussian blending function is introduced at the subspace boundaries. The purpose of this blending is to maintain continuity in magnitudes and slopes at the subspace boundaries. The Gaussian blending function is defined by 


$$
\begin{array}{ll}
b(x)=\exp \left\{-\left[\left(x-x_{u}\right)_{+} / \sigma_{x}\right]^{2}\right\} & \text { for upper bound blending } \\
b(x)=\exp \left\{-\left[\left(x-x_{l}\right)_{-} / \sigma_{x}\right]^{2}\right\} & \text { for lower bound blending }
\end{array}
$$

where $x_{u}$ and $x_{l}$ are the upper and lower limit values for explanatory variable $x$, and

$$
\begin{aligned}
& \left(x-x_{u}\right)_{+} \equiv\left\{\begin{array}{cc}
\left(x-x_{u}\right) & x>x_{u} \\
0 & x \leq x_{u}
\end{array}\right. \\
& \left(x-x_{l}\right)_{-} \equiv\left\{\begin{array}{cc}
\left(x-x_{l}\right) & x<x_{l} \\
0 & x \geq x_{l}
\end{array}\right.
\end{aligned}
$$

The Gaussian blending functions shown above multiply the updated response surface to fade it out, and the surrounding aerodynamic database to fade it in, as each explanatory variable takes values from the interior of the updated subspace toward the surrounding aerodynamic database. The blending functions are applied at each boundary of the subspace. The values of $x_{u}$ and $x_{l}$ for each explanatory variable are the subspace boundary values, determined from the flight maneuver(s).

The value of $\sigma_{x}$ was selected as

$$
\sigma_{x}=\left|x_{u}-x_{l}\right| / 10
$$

This choice for $\sigma_{x}$ was found to give reasonably smooth transitions. Larger values of $\sigma_{x}$ initiate the blending farther from the subspace boundaries, and therefore result in a more gradual transition. Smaller values of $\sigma_{x}$ restrict the range of influence of the blending to areas nearer the boundaries, but produce a more abrupt transition. Using the Gaussian blending functions ensures that there are no discontinuities in magnitude or slope at the subspace boundaries when using the updated response surface model.

\section{Demonstration using Flight Data}

For flight data analysis and modeling, the non-dimensional force and moment coefficients are computed by substituting measured and known quantities on the right sides of the following equations ${ }^{1}$

$$
\begin{gathered}
C_{X} \equiv-C_{A}=\frac{\left(m a_{x}-T\right)}{\bar{q} S} \quad C_{Y}=\frac{m a_{y}}{\bar{q} S} \quad C_{Z}=-C_{N}=\frac{m a_{z}}{\bar{q} S} \\
C_{D}=-C_{X} \cos \alpha-C_{Z} \sin \alpha \\
C_{l}=\frac{I_{x}}{\bar{q} S b}\left[\dot{p}-\frac{I_{x Z}}{I_{x}}(p q+\dot{r})+\frac{\left(I_{z}-I_{y}\right)}{I_{x}} q r\right] \\
C_{m}=\frac{I_{y}}{\bar{q} S \bar{c}}\left[\dot{q}+\frac{\left(I_{x}-I_{z}\right)}{I_{y}} p r+\frac{I_{x z}}{I_{y}}\left(p^{2}-r^{2}\right)\right] \\
C_{n}=\frac{I_{z}}{\bar{q} S b}\left[\dot{r}-\frac{I_{x z}}{I_{z}}(\dot{p}-q r)+\frac{\left(I_{y}-I_{x}\right)}{I_{z}} p q\right]
\end{gathered}
$$


This produces $N$ values of the non-dimensional force and moment coefficients, where $N$ is the number of data points. These values are often called measured force and moment coefficients, even though they not measured directly, but rather computed from other measurements and known quantities. Explanatory variables such as angle of attack, Mach number, pitch rate, and control surface deflections, are measured directly.

The flight test aircraft used for this demonstration was the NASA F-18 High Alpha Research Vehicle (HARV), shown in Figure 2. Table 1 contains geometry and mass properties for the F-18 HARV. Further information about this aircraft can be found in Ref. [9].

Figure 3 shows data from a longitudinal flight test maneuver flown on the F-18 HARV. The maximum and minimum values of the explanatory variables shown in Figure 3 defined the boundaries of the subspace to be interrogated in the aerodynamic data tables. The aerodynamic data tables come from wind tunnel data for the F/A-18 configuration, which differs somewhat from the F-18 HARV. The aerodynamic data tables are a simplified subset of the aerodynamic

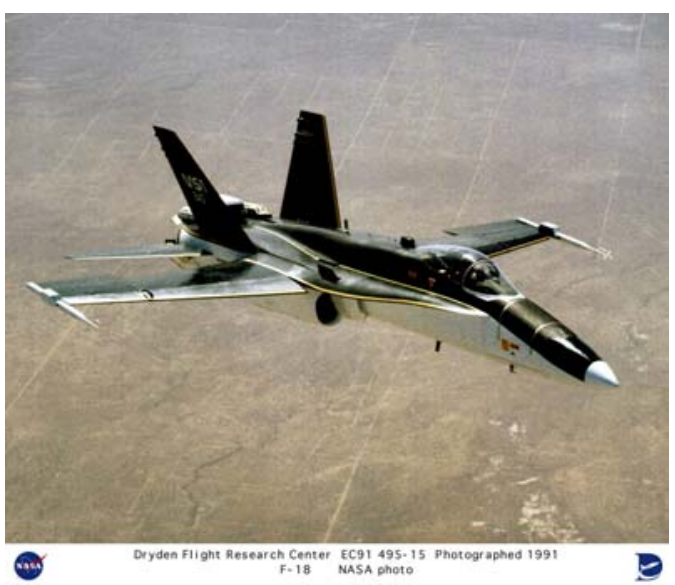

Figure 2. F-18 High Alpha Research Vehicle (HARV) database used for the simulation documented in Ref. [10]. The idea is to use flight data from the F-18 HARV to update the simplified aerodynamic database for the F/A-18 so that the updated model characterizes the aerodynamics of the F-18 HARV.

Data points for the initial response surface modeling were chosen from the wind tunnel aerodynamic database to encompass the range of explanatory variables observed in the flight data. The explanatory variables were angle of attack (6 values), Mach number (2 values), non-dimensional pitch rate (3 values), stabilator deflection (3 values), leading-edge flap deflection (2 values), and trailing-edge flap deflection (2 values). The aerodynamic data tables were interrogated at all possible combinations of the explanatory variable values, for a total of $6 \times 2 \times 3 \times 3 \times 2 \times 2$ or 432 data points. All of the data collected were actual data points (not interpolated values) from the aerodynamic data tables.

Based on the data collected from the aerodynamic database, response surface models were identified for drag coefficient, lift coefficient, and pitching moment coefficient using the multivariate orthogonal function method described earlier. The identified model structure, parameter values, and standard errors for the pitching moment coefficient response surface model are given in the first two columns of Table 2. Estimated parameter values are listed, with their standard errors given in parentheses below.

Using the response surface modeling results as a priori information, the flight data were analyzed as described previously. The resulting parameter estimates and standard errors are shown in the third column of Table 2. The fourth column contains the model parameter estimates obtained based on the flight data alone, i.e., using the same model structure, but without any a priori information.

The upper plot in Figure 4 shows the fit of the response surface model to the data collected from the aerodynamic database. The saw tooth appearance of the data and model fit is the result of sequentially plotting data from a rectangular hyper-grid. The lower plot shows the residual, which is the difference between the pitching moment coefficient from the aerodynamic data tables and the response surface model. The model captures most of the functional dependence, which is indicated by the nearly random character of the residual plot.

Figure 5a shows the result of predicting the F-18 HARV flight values of pitching moment coefficient using the model identified from the aerodynamic data tables for the F/A-18, exhibiting a significant mismatch. The flight-updated model was applied in the same way, with the result shown in Figure 5b. The match to the flight data is greatly improved, with the residuals more closely approximating a random sequence.

Finally, the flight-updated model was tested in a prediction case, on a different maneuver that was not used in the modeling. The explanatory variable ranges covered by the prediction maneuver exceeded the subspace boundaries for the original flight update. This was done purposely, to test the Gaussian blending at the subspace boundaries. Figure 6 shows the data for explanatory variables during the prediction maneuver. Using this data and the flight-updated model from before, the pitching moment coefficient was computed for the prediction maneuver. Figure 7 indicates that the flight-updated model predicts the pitching moment with approximately the same fidelity as was seen for the modeling data in Figure 5. Figures 8 and 9 show another prediction case, with similar results. 


\section{Discussion of Results}

The results shown in Table 2 and in Figures 5 through 9 indicate that the automated procedure described here was very effective for updating an aerodynamic database to incorporate information from flight data. Similar results were observed for the lift and drag coefficients. Results in Table 2 showed that the updating procedure worked as intended. For example, the estimate of $C_{m_{M}}$ from the aerodynamic database was much more accurate than the value from flight data alone, so the flight-updated model kept a value close to the estimate from the aerodynamic database model. The flight-updated estimate of $C_{m_{o}}$ was closer to the estimate from flight data alone, because the estimate from the aerodynamic database was less accurate. Similarly, the flight-updated estimate of $C_{m_{q}}$ was adjusted relative to the estimate from the a priori model based on the aerodynamic database, because both the a priori model and the flight data alone produced estimates of this parameter with comparable accuracy. Note that the model structure shown in Table 2 was not appropriate for the information content in the flight data; however, that model structure was still used for the modeling based on flight data alone, so that comparisons could be made to the model identified from the aerodynamic data tables and the flight-updated model. This fact causes a few unusual changes in the model parameters, because of shifting dependencies among model terms that should not be present for the modeling based on flight data alone. The good prediction capability demonstrated in Figures 7 and 9 support the idea that the flight-updated model properly characterizes the aerodynamic functional dependencies for the F-18 HARV aircraft.

The final step in the updating procedure is to incorporate the flight-updates into the aerodynamic database. This can be done by implementing increments to the original aerodynamic database. The increments only apply when the explanatory variables are within the subspace used for the flight update. Since the same model structure was retained throughout the analysis, the flight-update increment can be determined easily as a response surface model with parameter values equal to the difference between the parameter values for the a priori model identified from the aerodynamic database and the flight-updated model. In Table 2, this would be a model with the terms indicated in the first column,

$$
C_{m}=C_{m_{o}}+C_{m_{\delta_{s}}} \delta_{s}+C_{m_{\alpha}} \alpha+C_{m_{M}} M+C_{m_{\alpha M}} \alpha M+C_{m_{q}} \frac{q \bar{c}}{2 V_{o}}+C_{m_{\alpha^{2}}} \alpha^{2}+C_{m_{\delta_{f}}} \delta_{f}+C_{m_{\alpha \delta_{s}}} \alpha \delta_{s}(37)
$$

with parameter values equal to the difference between the flight-updated values in column 3 and the $a$ priori model values in column 2 . In this case, the nonlinearities that were identified automatically using the orthogonal function modeling approach would have been difficult to guess or to determine in another way. Of course, this parametric flight-update increment model could be interrogated for various values of the explanatory variables on the right side of Eq. (37) to produce tabular data for the update, if that was desired.

\section{Conclusion}

This work describes and demonstrates a method for rapidly and automatically modifying an aerodynamic database (which can be approximate) based on flight data, in order to accurately characterize aerodynamic functional dependencies and thereby achieve good aerodynamic prediction capability. The technique works by automatically combining the best information from both ground-based aerodynamic data and flight data, using statistical weighting determined from the data. A local response surface model is identified first, based on data collected from a subspace of the aerodynamic database, and using least-squares modeling with orthogonal modeling functions generated from the data. This identifies an adequate model structure with estimated model parameters and uncertainty bounds, and represents the a priori model for flight data analysis. A Bayesian approach to the least squares modeling provides an automatic and statistically-based mechanism for determining how much the a priori parameter values identified from aerodynamic database should be modified according to information in the flight data.

The method implements a common-sense approach of updating the aerodynamic model based on flight data when there is sufficient information to do so, but otherwise keeping the information embodied in the aerodynamic database. The decisions on balancing the contributions from each information source are made automatically, based on statistical principles. Finally, the identified flight updates are blended smoothly with the surrounding database using Gaussian blending functions. The capability demonstrated in this work can have significant impact on simulation fidelity improvement, flight test efficiency, and ground-based testing requirements. 


\section{Acknowledgments}

This work was supported in part by the U.S. Air Force under contract AF99-330.

\section{References}

${ }^{1}$ Klein, V. and Morelli, E.A., Aircraft System Identification - Theory and Practice, AIAA Education Series, AIAA, Reston, VA, 2006.

${ }^{2}$ Morelli, E.A. “Global Nonlinear Aerodynamic Modeling using Multivariate Orthogonal Functions,” Journal of Aircraft, Vol. 32, No. 2, March-April 1995, pp. 270-77.

${ }^{3}$ Morelli, E.A. and DeLoach, R., "Wind Tunnel Database Development using Modern Experiment Design and Multivariate Orthogonal Functions,” AIAA Paper 2003-0653, 41 $1^{\text {st }}$ AIAA Aerospace Sciences Meeting and Exhibit, Reno, NV, January 2003.

${ }^{4}$ Barron, A.R., "Predicted Squared Error : A Criterion for Automatic Model Selection,” Self-Organizing Methods in Modeling, Farlow, S.J., Ed., Marcel Dekker, Inc., New York, NY, 1984, pp. 87-104.

${ }^{5}$ Morelli, E.A. and DeLoach, R. "Response Surface Modeling using Multivariate Orthogonal Functions," $39^{\text {th }}$ AIAA Aerospace Sciences Meeting and Exhibit, AIAA Paper 2001-0168, Reno, NV, August 2001.

${ }^{6}$ Morelli, E.A. and Proffitt, M.S. "Modeling the High Speed Research Cycle 2B Longitudinal Aerodynamic Database using Multivariate Orthogonal Functions,” NASA CR-1999-209525, December 1999.

${ }^{7}$ Morelli, E.A. "Global Nonlinear Parametric Modeling with Application to F-16 Aerodynamics," ACC Paper WP04-2, American Control Conference, Philadelphia, PA, June 1998.

${ }^{8}$ Morelli, E.A., "Practical Aspects of the Equation-Error Method for Aircraft Parameter Estimation," AIAA-2006-6144, AIAA Atmospheric Flight Mechanics Conference, Keystone, CO, August 2006.

${ }^{9}$ Bowers, A.H. et al. “An Overview of the NASA F-18 High Alpha Research Vehicle,” NASA TM 4772, October 1996.

${ }^{10}$ Buttrill, C.S., Arbuckle, P.D., and Hoffler, K.D. “Simulation Model of a Twin-Tail, High Performance Airplane,” NASA TM 107601, April 1992.

Table 1 Geometry and mass properties for the F-18 HARV aircraft

\begin{tabular}{cc}
\hline \hline length $\bar{c}, \mathrm{ft}$ & 11.52 \\
\hline wing span $b, \mathrm{ft}$ & 37.42 \\
\hline wing area $S, \mathrm{ft}^{2}$ & 400 \\
\hline$x_{\text {ref }}, \mathrm{ft}$ & 458.56 \\
\hline$y_{\text {ref }}, \mathrm{ft}$ & 0.00 \\
\hline$z_{r e f}, \mathrm{ft}$ & 100.00 \\
\hline$x_{c g}$, in & 459.86 \\
\hline$y_{c g}$, in & -0.06 \\
\hline$z_{c g}$, in & 102.78 \\
\hline$m$, slugs & $1,006.8$ \\
\hline$I_{x}$, slugs- $\mathrm{ft}^{2}$ & 21,977 \\
\hline$I_{y}$, slugs $-\mathrm{ft}^{2}$ & 172,718 \\
\hline$I_{z}$, slugs $-\mathrm{ft}^{2}$ & 187,030 \\
\hline$I_{x z}$, slugs- $\mathrm{ft}^{2}$ & $-2,111$ \\
\hline \hline
\end{tabular}


Table 2 Pitching moment coefficient models

\begin{tabular}{|c|c|c|c|}
\hline $\begin{array}{l}\text { Model } \\
\text { Term }\end{array}$ & $\begin{array}{c}\text { F/A-18 } \\
\text { aerodynamic } \\
\text { database } \\
\hat{\theta} \\
(s)\end{array}$ & $\begin{array}{c}\text { Updated using } \\
\text { F-18 HARV } \\
\text { flight data } \\
\hat{\theta} \\
(s)\end{array}$ & $\begin{array}{c}\text { F-18 HARV } \\
\text { flight data } \\
\text { alone } \\
\hat{\theta} \\
(s)\end{array}$ \\
\hline$C_{m_{o}}$ & $\begin{array}{c}-3.245 \mathrm{e}-02 \\
(6.778 \mathrm{e}-04)\end{array}$ & $\begin{array}{c}-1.681 \mathrm{e}-02 \\
(1.324 \mathrm{e}-04)\end{array}$ & $\begin{array}{c}-1.502 \mathrm{e}-02 \\
(2.830 \mathrm{e}-04)\end{array}$ \\
\hline$C_{m_{\delta_{S}}}$ & $\begin{array}{l}-1.632 \mathrm{e}-02 \\
(8.747 \mathrm{e}-05)\end{array}$ & $\begin{array}{l}-1.644 \mathrm{e}-02 \\
(5.440 \mathrm{e}-05)\end{array}$ & $\begin{array}{l}-1.722 \mathrm{e}-02 \\
(1.377 \mathrm{e}-04)\end{array}$ \\
\hline$C_{m_{\alpha}}$ & $\begin{array}{l}-2.531 \mathrm{e}-03 \\
(1.805 \mathrm{e}-04)\end{array}$ & $\begin{array}{l}-5.657 e-03 \\
(9.446 e-05)\end{array}$ & $\begin{array}{r}-5.198 \mathrm{e}-03 \\
(3.957 \mathrm{e}-04)\end{array}$ \\
\hline$C_{m_{M}}$ & $\begin{array}{c}-8.358-02 \\
(2.142 \mathrm{e}-03) \\
\end{array}$ & $\begin{array}{l}-8.340 \mathrm{e}-02 \\
(2.132 \mathrm{e}-03)\end{array}$ & $\begin{array}{c}1.796 \mathrm{e}-01 \\
(3.291 \mathrm{e}-02) \\
\end{array}$ \\
\hline$C_{m_{\alpha M}}$ & $\begin{array}{c}-1.139 \mathrm{e}-02 \\
(5.808-04) \\
\end{array}$ & $\begin{array}{l}-1.130 \mathrm{e}-02 \\
(5.802 \mathrm{e}-04)\end{array}$ & $\begin{array}{c}6.182 \mathrm{e}-02 \\
(2.334 \mathrm{e}-02)\end{array}$ \\
\hline$C_{m_{q}}$ & $\begin{array}{c}-4.537 \mathrm{e}+00 \\
(2.430-01)\end{array}$ & $\begin{array}{l}-7.306 \mathrm{e}+00 \\
(1.694 \mathrm{e}-01)\end{array}$ & $\begin{array}{r}-1.688 \mathrm{e}+01 \\
(8.913 \mathrm{e}-01)\end{array}$ \\
\hline$C_{m_{\alpha^{2}}}$ & $\begin{array}{c}6.851 \mathrm{e}-04 \\
(3.977 \mathrm{e}-05)\end{array}$ & $\begin{array}{c}4.720 \mathrm{e}-04 \\
(3.100 \mathrm{e}-05)\end{array}$ & $\begin{array}{c}-1.140 \mathrm{e}-03 \\
(1.059 \mathrm{e}-04)\end{array}$ \\
\hline$C_{m_{\delta_{f}}}$ & $\begin{array}{c}1.616 \mathrm{e}-03 \\
(1.323 \mathrm{e}-04)\end{array}$ & $\begin{array}{c}9.579 \mathrm{e}-04 \\
(9.332 \mathrm{e}-05)\end{array}$ & $\begin{array}{c}5.499 \mathrm{e}-03 \\
(4.562 \mathrm{e}-04)\end{array}$ \\
\hline$C_{m_{\alpha \delta_{s}}}$ & $\begin{array}{c}2.688 \mathrm{e}-04 \\
(2.371 \mathrm{e}-05)\end{array}$ & $\begin{array}{c}9.244 \mathrm{e}-05 \\
(1.902 \mathrm{e}-05)\end{array}$ & $\begin{array}{c}3.073 \mathrm{e}-04 \\
(5.633 \mathrm{e}-05)\end{array}$ \\
\hline
\end{tabular}



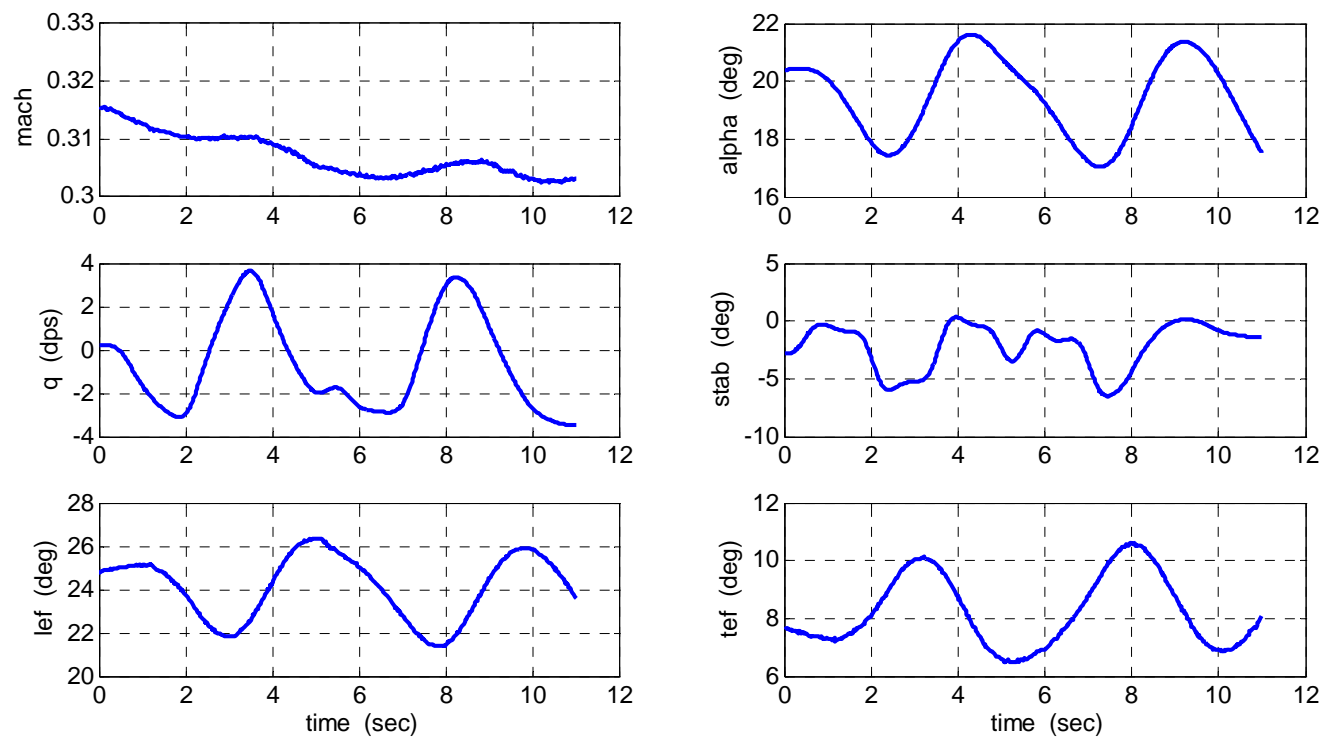

Figure 3. F-18 HARV flight data for a longitudinal maneuver
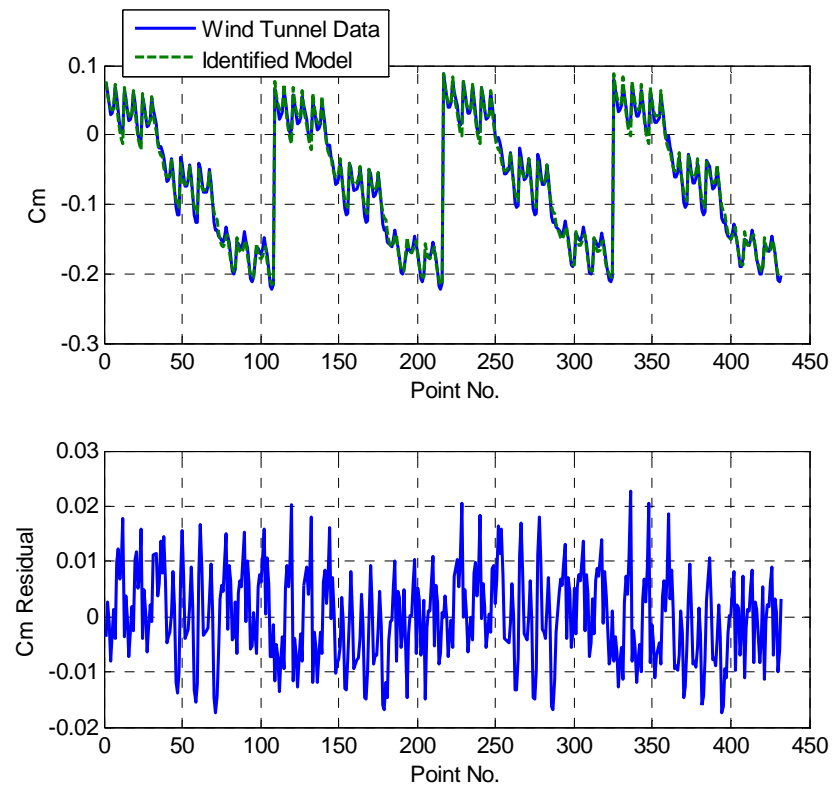

Figure 4. F/A-18 aerodynamic database response surface model fit 
a)
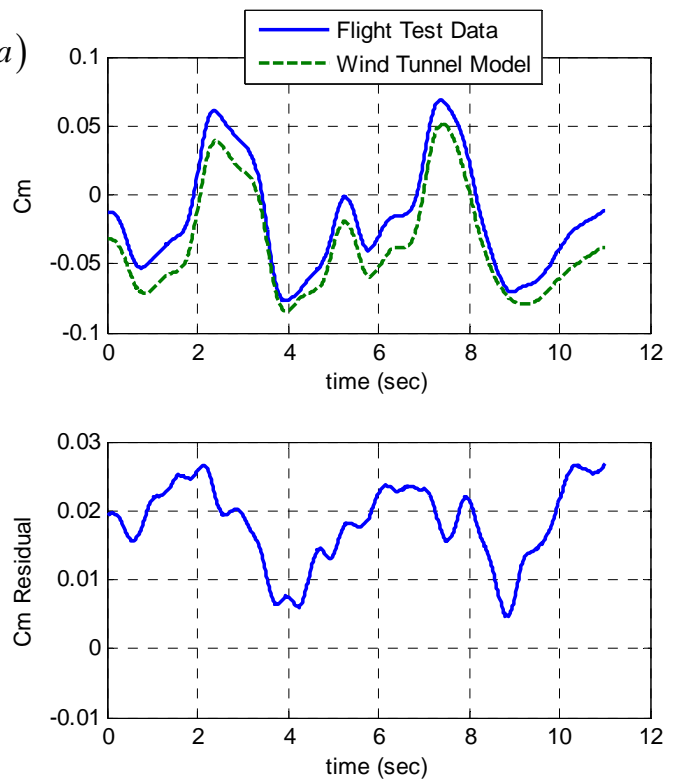

b)
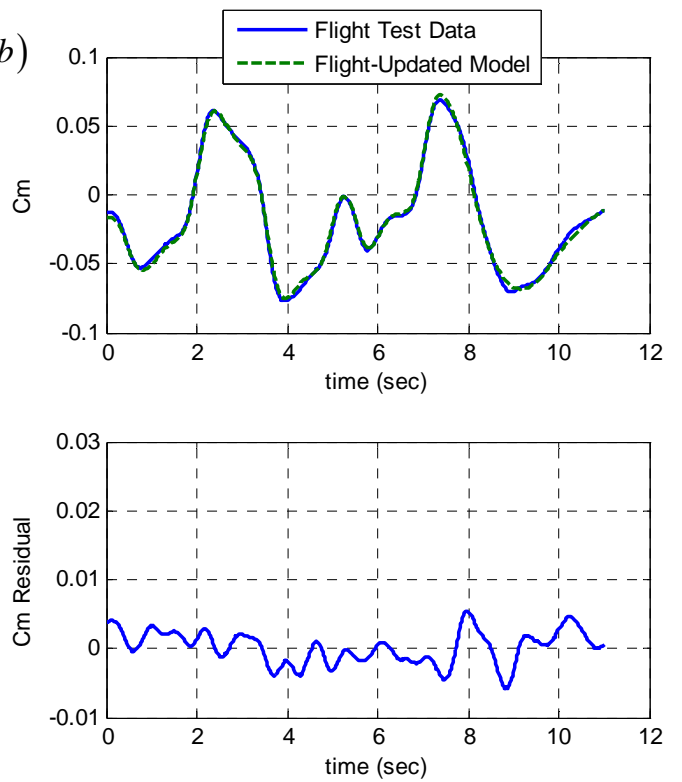

Figure 5. Model fit to F-18 HARV flight data using: $a$ ) F/A-18 wind-tunnel aerodynamic database and b) flight-updated response surface model 

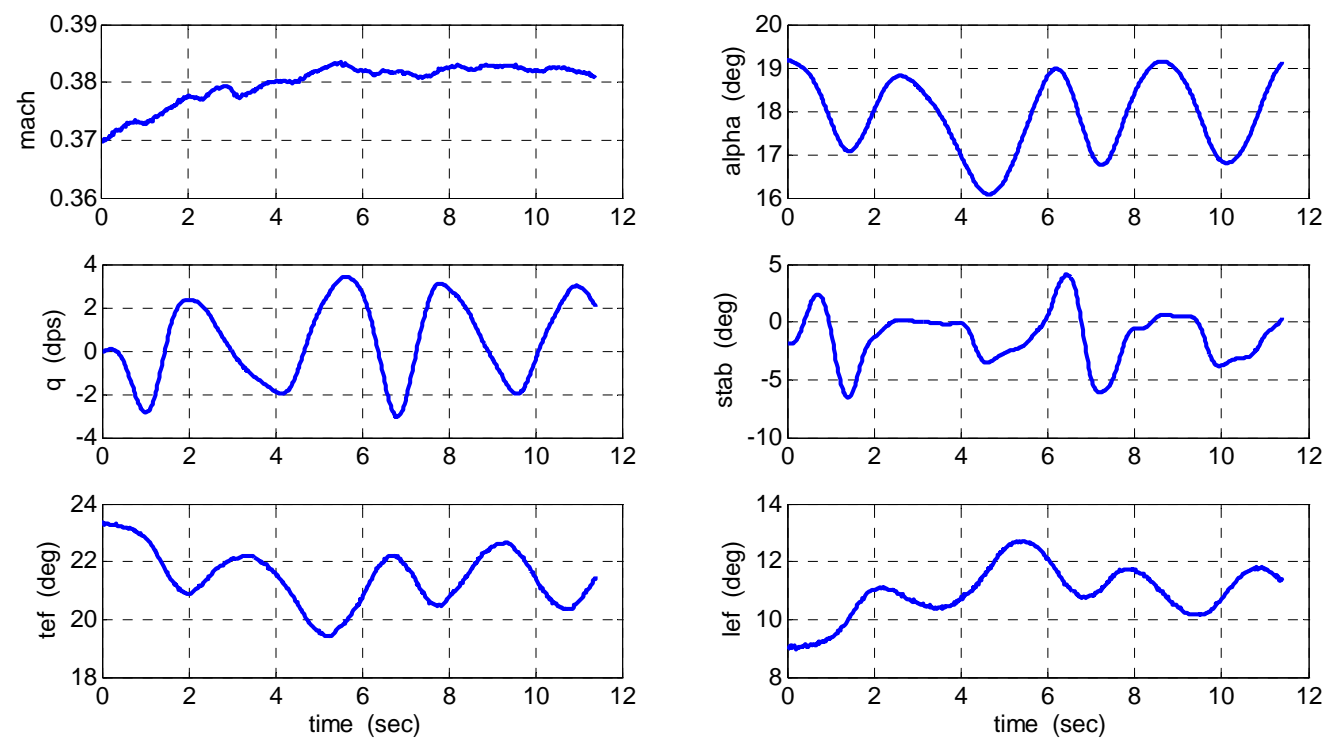

Figure 6. F-18 HARV flight data for a longitudinal prediction maneuver

a)
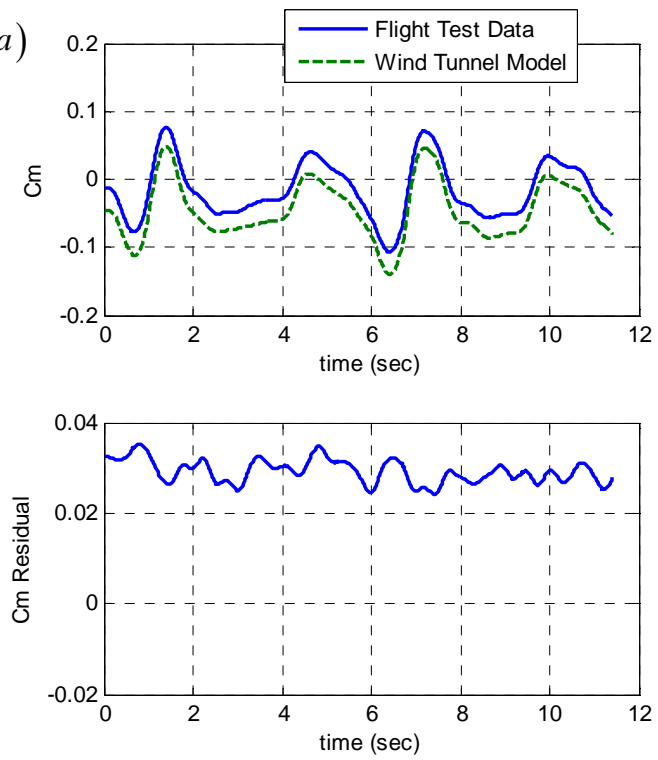

b)
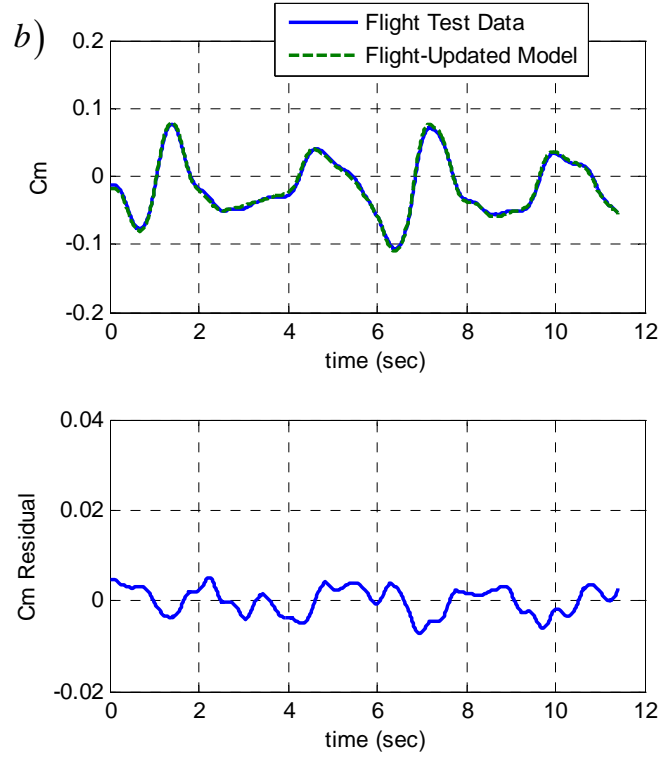

Figure 7. Prediction of F-18 HARV flight data using: $a$ ) F/A-18 wind-tunnel aerodynamic database and b) flight-updated response surface model using a flight-updated response surface model 

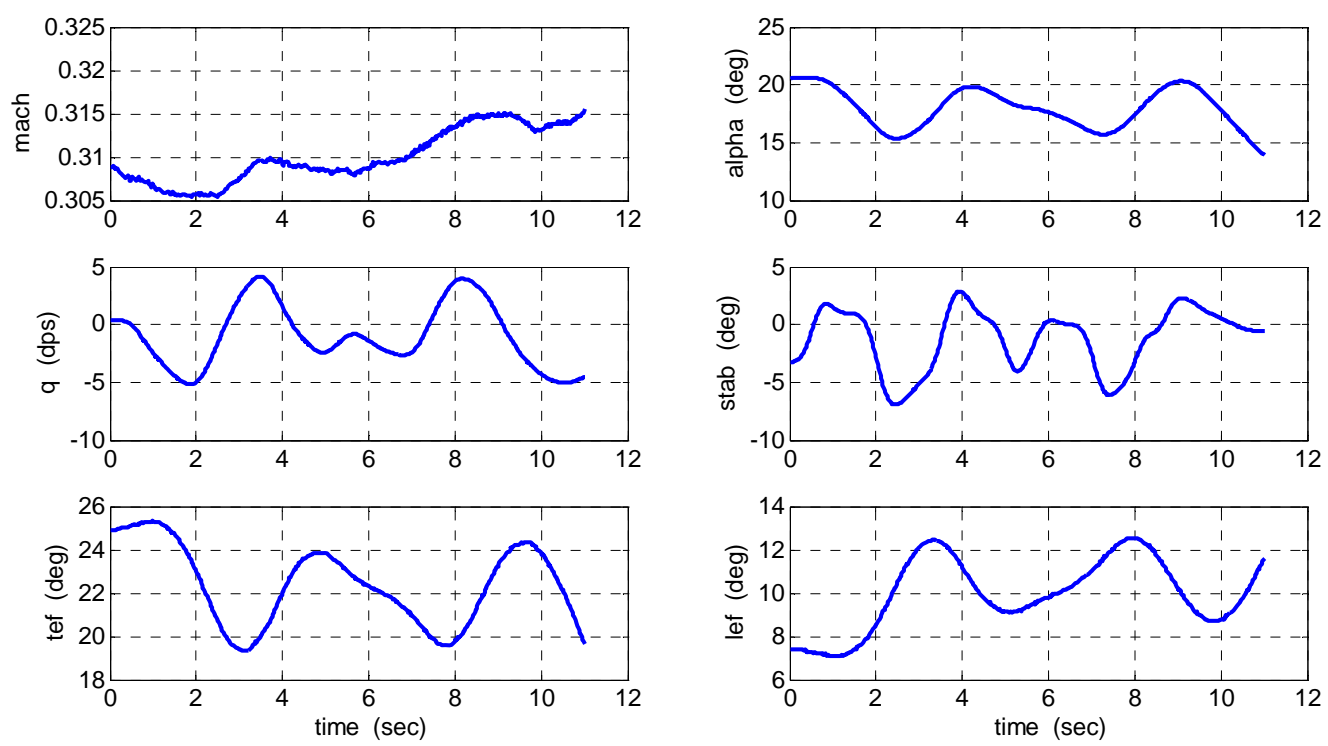

Figure 8. F-18 HARV flight data for a longitudinal prediction maneuver

a)
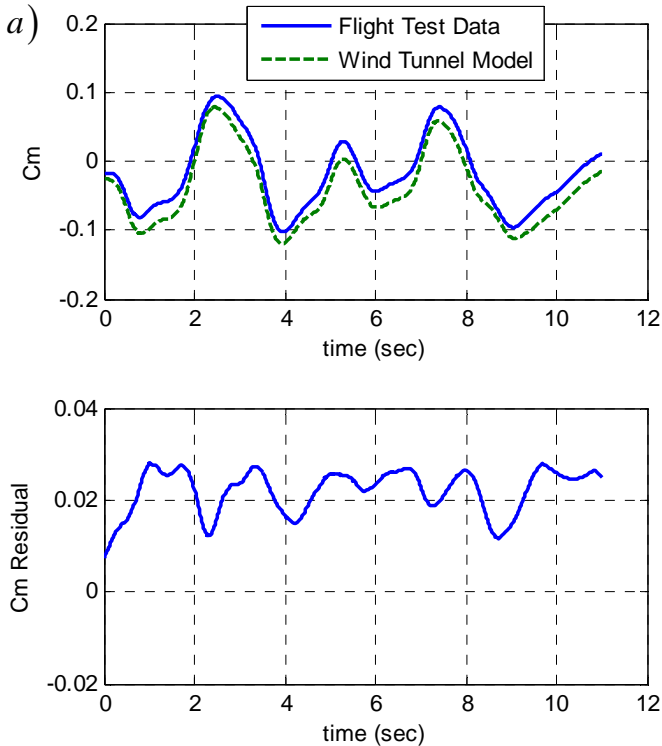

b)
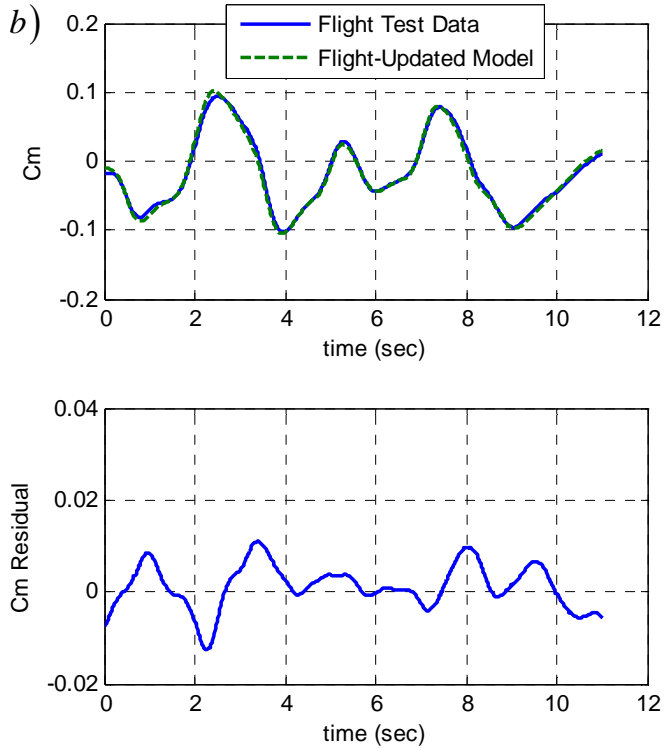

Figure 9. Prediction of F-18 HARV flight data using: $a$ ) F/A-18 wind-tunnel aerodynamic database and b) flight-updated response surface model 